Spiel und damit häufigste Einzelursache. Doch selbst hier war es nicht der Ball, sondern der gegnerische Körper, der die meisten Gehirnerschütterungen auslöste.

Gäbe es weniger Körperkontakt beim Fußball, würde also womöglich die Commotiogefahr sinken. Vorschläge dazu gibt es, ebenso wie die Idee, den Spielern Helme zu verpassen. Hier allerdings greift ein Lehrsatz, den der ehemalige Kapitän der Dortmunder Borussia Adi Preißler formuliert hat: „Grau ist alle Theorie - entscheidend ist auf dem Platz.“

\title{
Fußball-Forensik
}

Ob durch Kopfbälle oder gegnerische Köpfe: Die Gefahr fürs Gehirn, die vom Kicken ausgeht, ist offenbar real. Hamburger Rechtsmediziner haben SchädelHirn-Traumen von Sportlern untersucht [6]. Sie sind dabei auch auf Berichte über Fußballer mit chronischen bzw. kumulativen Hirnschäden gestoßen. Einen be sonderen Grund dafür, warum in einem sportlich gesunden Körper nicht immer ein gesundes Hirn wohnt, sehen sie im sogenannten Second-Impact-Syndrom. Gemeint ist damit ein zweites Trauma, das einen Athleten trifft, der die Folgen des ersten noch nicht vollständig auskuriert hat. Dies wirkt sich mitunter fatal

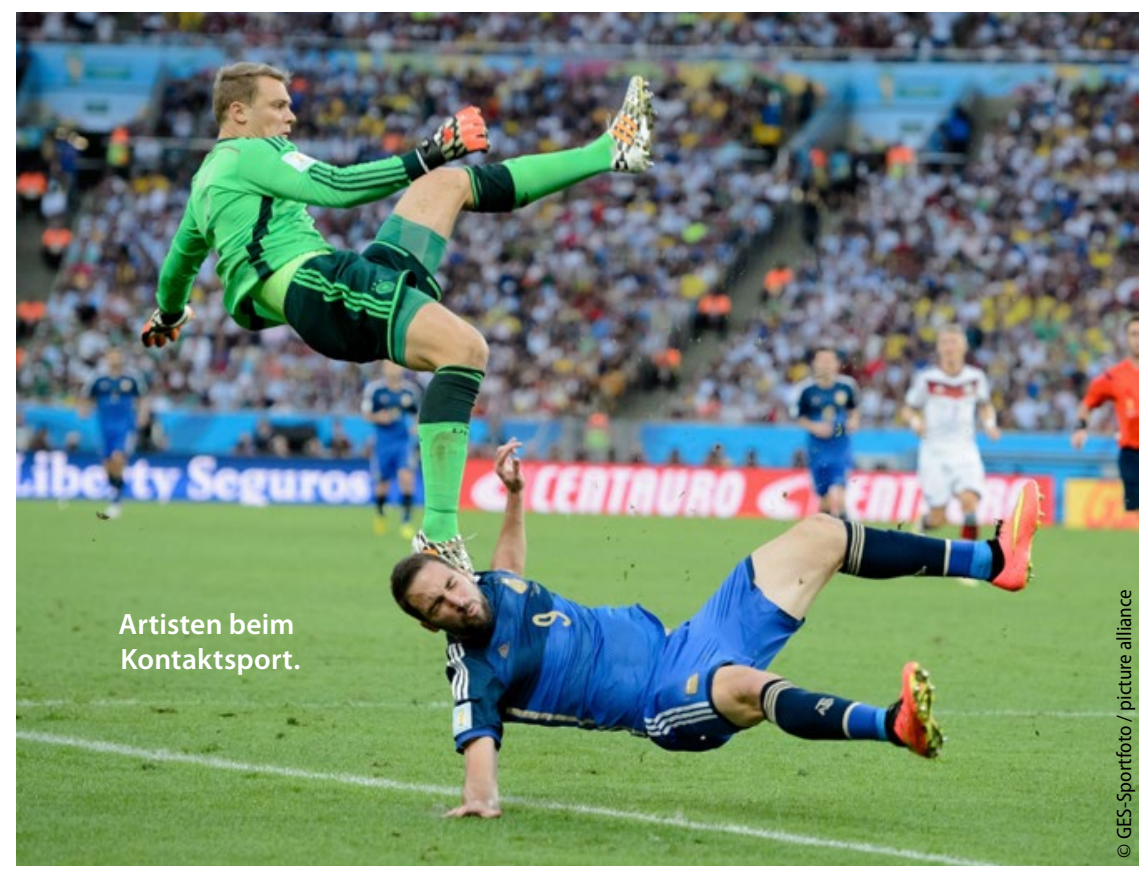

aus, selbst wenn das zweite Ereignis den Sportler nur mit vergleichsweise geringer Energie trifft. Schwere diffuse Traumata mit Bewusstlosigkeit und Hirnschwellung können die Folge sein, wobei vermutlich eine Störung der zerebralen Autoregulation die Ursache ist.

Spekulativ bleibt, ob nicht so mancher Fall von mutmaßlichem plötzlichem Herztod eines Athleten nach einer banalen Kollision mit einem Gegenspieler in Wahrheit auf ein Second-ImpactSyndrom zurückgeht. Die Hamburger

\section{Ischiokrurale Schwachstellen gezielt stärken}

Verletzungen der rückwärtigen Oberschenkelmuskulatur sind die häufigsten Muskelverletzungen im Fußball. In welcher Weise eine Übung namens "Nordic Hamstring" (Nordic Hamstring Exercise, NHE) die Häufigkeit von Läsionen der ischiokruralen Muskulatur (IKM) beeinflusst, haben Sport- und Rehabilitationsmediziner der Universität Utrecht mit insgesamt 40 Amateurfußballmannschaften untersucht [Am J Sports Med 2015;43:1316-23]. 20 Teams mit 292 Spielern bauten die Übung nach der Winterpause 2012/2013 für 13 Wochen in ihren Trainingsplan ein. Geübt wurde NHE zwei- bis dreimal pro Trainingseinheit und dabei jeweils fünf- bis zehnmal wiederholt. Die 20 anderen Teams mit 287 Spielern trainierten wie gehabt.

Für NHE kniet Spieler A in aufrechter Haltung, während Spieler B die Fersen und Unterschenkel von A auf die Unterlage drückt. A lässt sich so langsam wie möglich nach vorne sinken, wobei er Oberkörper und Oberschenkel in Streckung hält. Die exzentrische Belastung der rückseitigen Oberschenkelmuskulatur - Spannung bei gleichzeitiger Streckung - wird maximiert. Schließlich bremst A den Fall mit Händen und Armen, stößt sich, nachdem der Brustkorb den Boden berührt hat, vom Untergrund ab und geht zurück in die Ausgangstellung. (Ein Video zur Übung findet sich im Internet unter https://www.youtube.com/watch?v=lbmuE4clhg4.)

Interventions- und Kontrollgruppe unterschieden sich signifikant in der Häufigkeit von IKM-Verletzungen. Die NHE-Gruppe hatte eine Inzidenz von 0,25/1000 Spielerstunden zu verzeichnen, in der Kontrollgruppe erreichte die Rate 0,8/1000. Das Risiko, solche Läsionen zu erleiden, sank durch NHE um rund $70 \%$.

$\mathrm{rb}$

Forensiker fordern eindringlich, Sportlern nach einem Schädel-Hirn-Trauma, auch nach einer vermeintlich geringfügigen Gehirnerschütterung, ausreichend Zeit zur Erholung zu geben.

Dass Fußballspiele nicht zuletzt mit dem Hirn entschieden werden, wusste auch der eingangs zitierte Bill Shankly. „Der Erfolg hängt beim Fußball stark vom Verstand ab“, sagte er. „Du musst daran glauben, dass du der Beste bist, und dann dafür sorgen, dass es wirklich so ist." Wie sich aber die Intelligenz eines Kickers über dessen Körper verteilt, darüber hatte Shankly eigene Vorstellungen. Einem Nachwuchsspieler, mit dem er wohl nicht ganz zufrieden war, beschied er: „Junge, das Problem mit dir ist: Dein ganzer Grips ist im Kopf.“

\section{Dr. Robert Bublak}

- 1. Junge A. Football injuries during FIFA tournaments and the Olympic Games, 1998-2001. Am J Sports Med 2004;32 (Suppl 1): S80-9

- 2. Waldén M et al. ACL injuries in men's professional football: a 15-year prospective study on time trends and return-to-play rates reveals only $65 \%$ of players still play at the top level 3 years after $A C L$ rupture. Br J Sports Med 2016, online 31. März; doi: 10.1136/bjsports-2015-095952

- 3. Carlin Cet al. Match Injuries in Professional Soccer: Interseasonal variation and effects of competition type, match congestion and positional role. Int J Sports Med 2010;31:271-6

- 4. Downs DS, Abwender D. Neuropsychological impairment in soccer athletes. J Sports Med Phys Fitness 2002;42:103-7 - 5. Rodrigues $A C$ et al. Effects of soccer heading on brain structure and function. Front Neurol 2016, online 21. März; doi: 10.3389/fneur.2016.00038

- 6. Matschke J et al. Schädel-Hirn-Trauma und Sport. Rechtsmedizin 2011;21:191-6 